\title{
Static Lightpath Establishment in Multilayer Traffic Engineering Under Physical Layer Impairments
}

\author{
Namik Sengezer and Ezhan Karasan
}

\begin{abstract}
This paper investigates the lightpath establishment problem in the optical layer, which arises in multilayer traffic engineering. The static version of the problem is considered, in which the set of requested lightpaths is known initially, and the objective is to set up as many of these lightpaths as possible by assigning the physical layer resources efficiently. In establishing the lightpaths, physical layer impairments are also considered, such that the bit error rates of all established lightpaths should remain within acceptable limits. A heuristic algorithm, called ROLE, is proposed for the solution of this problem. An integer linear programming (ILP) formulation for the static lightpath establishment with physical layer impairments is also developed, which considers the aggregated effect of physical layer impairments. This formulation is used as a performance benchmark for ROLE on smaller-size networks. It also provides upper bounds even when the ILP model cannot be solved to optimality. The performance of ROLE is close to the optimum solutions for all studied problem sets, for which the optimum solution can be obtained. ROLE significantly outperforms previously proposed algorithms in terms of the number of lightpaths established. The effect of switch cross-talk ratio on the problem solution is also investigated.
\end{abstract}

Index Terms-Lightpath establishment; Optical network; Physical layer impairments; Routing and wavelength assignment (RWA); Wavelength division multiplexing (WDM).

\section{INTRODUCTION}

M ost of today's Internet traffic is IP originated, and with the migration of many real-time and quality-of-service- (QoS-) requiring services to IP, IP is foreseen to continue being the dominant network layer protocol in the future. The highly varying nature

Manuscript received December 17, 2009; revised June 7, 2010; accepted July 7, 2010; published August 9, 2010 (Doc. ID 121478).

The authors are with the Department of Electrical and Electronics Engineering, Bilkent University, TR-06800 Ankara, Turkey (e-mail: namik@ee.bilkent.edu.tr; ezhan@ee.bilkent.edu.tr)

Digital Object Identifier 10.1364/JOCN.2.000662 of the IP traffic and the QoS requirements of the services carried over IP urge telecom network operators to develop more cost-effective and efficient solutions rather than simply overprovisioning resources. Traffic engineering (TE) is one of the solutions that lead to flexible, quality-of-service providing, and cost-efficient networks [1].

Traffic engineering is typically performed in IP networks today by deploying multiprotocol label switching (MPLS) under the IP layer [2]. MPLS provides powerful functionalities for traffic engineering, such as bandwidth-guaranteed connections and explicit routing capability $[3,4]$. The dominant transport layer technology in today's metro and backbone networks is wavelength division multiplexing (WDM). By dividing the bandwidth capacity of a single fiber into multiple channels and by providing all-optical channels, WDM can eliminate the need for expensive optical-toelectronic conversions and make more efficient use of fiber's transmission capacity.

This multilayered network structure raises the questions of on which layer(s) traffic engineering should be applied and how. There are three commonly accepted models proposed by the Internet Engineering Task Force (IETF) for the interoperation of the IP/ MPLS and optical layers: overlay, peer, and augmented [5]. In the overlay model, the control planes of the two layers are separated. In the peer model, a single control plane is deployed over the IP/MPLS and optical layers, where the joint control plane controls both layers. In the augmented model, routing on each layer is controlled by the control plane of the corresponding layer, but certain information can be shared between the control planes.

Among these models, the overlay model is easier to implement with today's technology and can be preferred by the operators to ensure the privacy of each layer [6]. In this model, resource optimization is carried out separately in the electronic and optical layers, and the layers interact in a client/server relationship. The electronic layer requests lightpaths from the optical layer, and establishment of these lightpaths on 
the physical topology is carried out by the optical layer control plane. The set of established lightpaths forms the virtual topology seen by the electronic layer, and the electronic layer routes the traffic demands on this virtual topology [7].

The problem of establishing the lightpaths requested by the electronic layer and assigning the physical layer resources to the established lightpaths is called the lightpath establishment problem. The solution of this problem is a part of multilayer traffic engineering (MTE) within the optical layer. The optical layer control plane decides which of the requested lightpath demands should be established and assigns a route and a wavelength to each of these lightpaths.

In this study, we investigate the static version of the lightpath establishment problem. In this problem, the set of requested lightpaths is known beforehand and the objective is to establish as many of the lightpaths from this set as possible, using limited physical layer resources. It is assumed that wavelength conversion is not available in the optical layer, and the wavelength continuity constraint should be satisfied along the established lightpaths. The physical layer impairments are also taken into consideration, so that bit error rates (BERs) along all established lightpaths should remain below the upper threshold.

We propose a polynomial time heuristic solution for the static lightpath establishment problem: reordered lightpath establishment (ROLE). We investigate the performance of ROLE and compare it with a recently proposed heuristic algorithm, pre-ordering least impact offline routing and wavelength assignment (POLIO-RWA) [8]. We also develop an integer linear programming (ILP) formulation for the static lightpath establishment problem subject to physical layer impairments. In the developed formulation, the aggregated effect of physical impairments is considered and hard constraints are imposed to guarantee that BERs along established lightpaths are below the chosen threshold. This ILP formulation is used as a performance benchmark for ROLE on smaller-size networks and for providing upper bounds even when the ILP model cannot be solved to optimality for larger topologies.

Our numerical studies show that ROLE can establish up to $14 \%$ more lightpaths than the POLIO-RWA algorithm. In our comparisons with ILP solutions, the number of lightpaths that can be established by ROLE is close to the optimum solutions for all the problem sets, for which the optimum solution can be obtained. In the ILP formulation solutions, longer lightpath demands have considerably higher blocking probabilities than shorter lightpaths. ROLE can produce solutions with a more fair blocking distribution according to lightpath demand lengths, in which longer lightpaths have a higher probability of being established, compared with the ILP formulation solutions. We also investigated the effect of the switch cross-talk ratio on the produced solutions. According to the results, for values smaller than $-35 \mathrm{~dB}$, the switch cross-talk ratio has a small effect on the number of established lightpaths and at $-25 \mathrm{~dB}$ it reaches its maximum effect.

The rest of the paper is organized as follows. Section II introduces the lightpath establishment problem under physical layer impairments and gives an overview of the related literature. The physical layer model is described in Section III. Section IV presents the developed ILP formulation for the investigated lightpath establishment problem. The proposed ROLE algorithm is presented in Section V. Numerical results and evaluation of the ILP and heuristic solutions are presented in Section VI, and Section VII concludes the paper.

\section{LIGHTPATH ESTABLISHMENT UNDER PHYSICAL IMPAIRMENTS}

For a given set of lightpath connection demands, routing and assigning a wavelength to each connection is called the routing and wavelength assignment (RWA) problem $[9,10]$. With no all-optical wavelength conversion capability, RWA is subject to two constraints: a lightpath should be assigned the same wavelength on all the links along its path, and no two lightpaths should be assigned the same wavelength on the same fiber. When the connection demands are permanent or long term, and known in advance as assumed in this study, the lightpath establishment problem is referred to as the static lightpath establishment problem, which can be solved by using offline RWA algorithms. On the other hand, when the lightpath demands arrive dynamically at random times and are served one at a time, the problem is referred to as the dynamic lightpath establishment problem, which should be solved using online RWA schemes. Linear programming formulations and heuristic search algorithms applying metaheuristics are proposed for the NP-complete offline RWA problem, whereas the online version is generally dealt with using fast heuristic algorithms. A detailed survey of various RWA approaches in the literature can be found in [10].

Finding a route with a free wavelength is not the only constraint when establishing a lightpath. As the optical signal propagates over a lightpath, it experiences quality degradation due to physical impairments in various components. Important physical impairments include amplified spontaneous emission (ASE) noise, chromatic dispersion, polarization mode dispersion (PMD), cross-talk, filter concatenation, 
self-phase modulation, cross-phase modulation, fourwave mixing, stimulated Raman scattering, and stimulated Brillouin scattering [11,12]. This signal quality degradation is cumulative, and, when the signal reaches the receiver node, the resulting BER may be above the acceptable value to be able to successfully communicate through the lightpath.

The RWA problem under physical layer impairments has been recently subject to increasing attention in the literature. The proposed approaches include linear programming formulations [13-15], applications of metaheuristics such as Tabu search [16], genetic algorithms [17,18], and heuristic algorithms [8,19-25]. A comprehensive literature survey on physical layer impairment-aware (PLIA) RWA solutions for both offline and online versions of the problem is presented in [26]. As stated in [8,15], most of the studies in the literature focus on online PLIA RWA and the more difficult offline version is rather less investigated. Below, we provide a brief review of recent studies on and various solution approaches to the offline PLIA RWA problem.

Linear-programming-based solutions have been considered for the PLIA RWA problem. In [13], the physical impairments are considered by assigning costs to links for each type of impairment. Then, the total flow cost in the network is minimized by using linear programming. Impairment effects depending on the interference of lightpaths, such as cross-talk, are neglected in that phase. In the next phase, BERs of the lightpaths to be established are calculated, and, if some BERs violate the minimum threshold, RWA is redone. LP formulations are also proposed in $[14,15]$. In these studies, the aggregated effect of different types of impairments is considered while taking the interference among the lightpaths into account. In [14], instead of using hard constraints, the physical layer impairments are taken into account by adding surplus variables in the objective function, whereas in [15] the aggregate effect of the physical layer impairments is included as a hard constraint. In both studies, ILP formulations are solved using linear programming relaxations with a piecewise linear cost function designed to produce integer solutions without integrality constraints, which is reported to yield integer solutions in most of the investigated problems. The formulation in [15] is compared with a non-PLIA RWA formulation and another formulation assuming the worst-case scenario for lightpath interference and shown to perform better than both in terms of blocking ratio and the number of wavelengths needed to satisfy the connection requests.

Use of metaheuristics is another approach used for solving the PLIA RWA problem. In [18], the authors propose a multiobjective optimization strategy. To constrain the physical layer impairments, parameters such as mean number of common hops, mean path length, and mean number of common edges are included in the objective function and genetic algorithms are used for the solution.

There are also studies applying heuristic RWA algorithms with a specific order. A sequential algorithm based on random search (RS) is proposed in [19]. The lightpath demands are considered one by one in a predefined order and served by using alternate routing with $k$ shortest paths and the first fit wavelength assignment scheme, without consideration of physical layer impairments. This process is repeated with random orderings of the demands and the order with the minimum number of blocked lightpath demands is chosen. After the RWA phase, a BER test is applied to each of the lightpaths in the solution set and a regenerator is placed along the lightpaths failing the test.

The POLIO-RWA algorithm [8] is the closest approach in the literature to our proposed solution. The lightpath demands are sorted according to the lengths of the shortest paths between their sources and destinations in an increasing order and served in that order. For each lightpath, the route and wavelength is chosen to maximize the minimum $Q$ factor among the active lightpaths. POLIO-RWA is compared with the solutions proposed in $[13,19]$ and is shown to have a lower blocking rate. However, in this approach RWA is applied with a fixed order and whether the performance could be further improved by rearranging the initial demand sorting is not investigated. The fairness of such an approach is another issue, since it is expected to block lightpath requests with longer paths.

In this study, we propose a sequential heuristic algorithm for the solution of the lightpath establishment problem: reordered lightpath establishment (ROLE). ROLE can be incorporated with various RWA algorithms, among which we implemented and tested the most commonly used ones. Applying rerouting and investigating different demand sorting combinations, it outperforms other heuristic algorithms proposed for the same problem. We have shown that ROLE achieves a better blocking performance than the POLIO-RWA algorithm. ROLE has a polynomial time complexity, and it is shown to have a performance close to the optimum solutions for all the studied cases for which the optimum solution can be obtained. It can produce fair solutions in terms of the distribution of the blocking ratio according to the lightpath demand lengths.

\section{PHYSICAL IMPAIRMENTS MODEL}

We use the optical wavelength routing node (WRN) architecture introduced in [20]. The WRN includes a 
cross-connect switch (XCS), a pair of erbium-doped fiber amplifiers (EDFAs), and optical power taps for monitoring purposes on the input and output sides at each port. The XCS consists of an array of demultiplexers, optical wavelength routing switches (WRSs), and an array of multiplexers.

The lightpath architecture is as presented in [27]. Along the fiber, backward-pumped distributed Raman amplifiers (DRAs) are used as in-line amplifiers. In each amplification span, a standard single-mode fiber (SSMF) is followed by a dispersion-compensating fiber (DCF). The attenuation along the DCF and SSMF and the chromatic mode dispersion along the SSMF are completely compensated by the DRA and the DCF, respectively. The attenuation between the last DRA and the WRN is compensated by the EDFAs at the input and output ports of the XCS along with the internal losses of the WRN resulting from the taps, multiplexers, demultiplexers, and switching fabric of the XCS.

The BER estimation is carried out by using the $Q$-factor approach given the following formulas [11]:

$$
\begin{aligned}
\mathrm{BER} & =\frac{1}{2} \operatorname{erfc}\left(\frac{Q}{\sqrt{2}}\right) \approx \frac{e^{-Q^{2} / 2}}{Q \sqrt{2 \pi}}, \\
Q & =\frac{I_{1}-I_{0}}{\sigma_{1}+\sigma_{0}} .
\end{aligned}
$$

Decomposing the impairment sources as eye related and noise related, the $Q$ factor at the destination node of the lightpath can be written as [28]

$$
\begin{aligned}
Q_{\text {end }}= & \frac{I_{1, \text { start }}-I_{0, \text { start }}}{\sigma_{1, \text { start }}+\sigma_{0, \text { start }}} \times \frac{\sigma_{1, \text { start }}+\sigma_{0, \text { start }}}{\sigma_{1, \text { end }}+\sigma_{0, \text { end }}} \\
& \times \frac{I_{1, \text { end }}-I_{0, \text { end }}}{I_{1, \text { start }}-I_{0, \text { start }}} .
\end{aligned}
$$

The indices start and end are used to denote the parameter values at the start and end of the line. $I_{0}$ and $I_{1}$ denote the current levels, and $\sigma_{0}$ and $\sigma_{1}$ are the noise standard deviations corresponding to bits 0 and 1, respectively. The last multiplicand in Eq. (3) is the eye-related penalty; the second multiplicand is the noise-related penalty; and the first multiplicand is $Q_{\text {start }}$, the initially assumed $Q$ factor. Eye-related and noise-related penalties can be calculated separately. Since CMD is compensated by the CDFs along the fiber as mentioned before, the eye-related penalty is due to PMD only, and it can be calculated as [28]

$$
Q_{P M D}=10.2 B^{2} D_{p}^{2} L
$$

where $B$ is the bit rate, $D_{p}$ is the PMD coefficient, and $L$ is the length of the lightpath. The sources for the noise-related penalty are ASE noise at the EDFAs and intrachannel node cross-talk. Other impairments such as FWM and interchannel cross-talk are disregarded in this model. The noise variance at the receiver can be written as the sum of four components [20]:

$$
\sigma_{m}^{2}=\sigma_{s x m}^{2}+\sigma_{s s p m}^{2}+\sigma_{s h m}^{2}+\sigma_{t h}^{2},
$$

where $m$ stands for the transmitted bit (0 or 1 ), and $\sigma_{s x m}^{2}, \sigma_{s s p m}^{2}, \sigma_{s h m}^{2}$, and $\sigma_{t h}^{2}$ are the variances of the signal cross-talk beat noise, ASE beat noise, shot noise, and thermal noise at the photodetector, respectively. These values depend on the signal, ASE noise, and cross-talk power levels at the destination node and can be calculated using the equations given in [20]. Using Eq. (3), the upper limit on the noise standard deviation along a lightpath can be written as

$$
\sigma_{\lim }=\frac{Q_{\text {start }} \times\left(\sigma_{1, \text { start }}+\sigma_{0, \text { start }}\right)}{Q_{\text {lim }}} \times Q_{P M D},
$$

where $Q_{\text {lim }}$ is the $Q$ factor corresponding to the maximum acceptable BER, and $\sigma_{0 \text {,start }}$ and $\sigma_{1, \text { start }}$ can be obtained from $Q_{\text {start }}$ and the channel power at the transmitters [21]. Then, for a lightpath to be established, we can define an upper bound on the noise standard deviation as an acceptance criteria as follows:

$$
\sigma_{1, \text { end }}+\sigma_{0, \text { end }} \leqslant \sigma_{\text {lim }} .
$$

The lightpaths satisfying Eq. (7) are guaranteed to have a BER lower than or equal to the acceptable threshold.

\section{ILP FORMULATION}

In the considered static lightpath establishment problem, the lightpath demands are given by a set $D$ and the objective is to route as many of these demands as possible using the provided network resources. This problem can be formulated using ILP, and the optimization problem is shown to be NP-complete even when the physical layer impairments are not considered [10].

In this section, we develop an ILP formulation for the lightpath establishment problem in the presence of physical layer impairments. The formulation is based on a path-based approach. Instead of applying constraints or assigning costs for different types of impairments separately, as in some of the previous studies, e.g., [13,14], the aggregate effect of physical layer impairments are considered in the constraints and the BER on the lightpaths in the solution is guaranteed to be below the acceptable threshold.

In the proposed formulation, the directed graph $G$ $=(N, L)$ represents the network topology with $N$ being the set of nodes and $L$ being the set of optical links. A set of paths are generated on the physical topology for each source-destination node pair, and the lightpath 
demands are routed on the paths chosen from these sets. Let $P_{d}$ denote the set of candidate paths along which demand $d \in D$ can be routed. The path and the wavelength used by demand $d$ is represented by the decision variable $x_{w d p}$ as follows:

$$
x_{w d p}=\left\{\begin{array}{ll}
1, & \text { if demand } d \text { is routed on path } p \text { at } w \\
0, & \text { otherwise }
\end{array},\right.
$$

where $p \in P_{d}$ and $w \in W$, and $W$ is the set of wavelengths. The ILP formulation maximizing the number of routed lightpath demands is given by

$$
\max \sum_{d \in D} \sum_{p \in P_{d}} x_{w d p}
$$

subject to

$$
\begin{gathered}
\sum_{w \in W} \sum_{p \in P_{d}} x_{w d p} \leqslant 1, \quad \forall d \in D, \\
\sum_{d \in D} \sum_{p \in P_{d}} x_{w d p} \times T_{l d p} \leqslant C_{l}, \quad \forall l \in L, \quad \forall w \in W .
\end{gathered}
$$

In this formulation, $C_{l}$ denotes the number of fibers on link $l$ and $T_{l d p}$ is an element of the link-path incidence matrix where

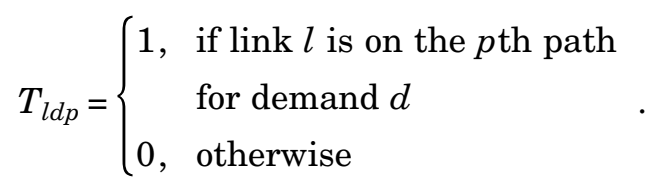

Constraint (8) implies that a demand can be routed on a single path and a single wavelength, and Eq. (9) represents the link capacity constraints.

To generate the constraints on the BER, we made use of Eq. (7), which gives an upper limit on the noise standard deviation for an acceptable BER. The noise variance at the receiver can be written as a function of signal, cross-talk, and ASE noise powers at the receiver, as proposed in [20]. Using the recursive equations given in [20], we generate the linear expressions $P_{S}(w, d, p), P_{A}(w, d, p)$, and $P_{X}(w, d, p)$, which represent the signal, ASE noise, and cross-talk power levels corresponding to lightpath demand $d$ at the receiver if it is routed on the $p$ th path in its path set and on wavelength $w$. Numerical values of $P_{S}(w, d, p)$ and $P_{A}(w, d, p)$ are calculated offline for each decision variable $x_{w d p}$; however, $P_{X}(w, d, p)$ depends on the routes of other lightpaths and its closed form expression contains the decision variables whose paths share at least one common node with the current lightpath candidate. The resulting linear expression derived from Eq. (5) for the noise variances at the receiver is as follows:

$$
\begin{aligned}
\sigma_{0}^{2}+\sigma_{1}^{2}= & \sum_{m \in\{0,1\}}\left(2 \xi_{p o l} R_{\lambda}^{2} b_{m} P_{S}(w, d, p) P_{X}(w, d, p)\right. \\
& +2 q R_{\lambda} B_{e}\left(b_{m} P_{S}(w, d, p)+P_{X}(w, d, p)\right. \\
& \left.+P_{A}(w, d, p)\right)+4 R_{\lambda}^{2} b_{m} P_{S}(w, d, p) \\
& \left.\times P_{A}(w, d, p) B_{e} / B_{o}+n_{t h} B_{e}\right) .
\end{aligned}
$$

The variable $b_{m}$ in Eq. (10) represents the ratio of the received optical signal power for the corresponding bit $m \in\{0,1\}$ to the average value of the received optical signal power. $b_{m}=0$ for $m=0$, and $b_{m}=2$ for $m$ $=1$. The remaining parameters are listed in Table I. The rest of the parameters used in the model are the same as in [20].

Equation (10) provides a linear expression for the noise variances; however, the condition to attain the target BER is that the sum of noise standard deviations for bits 0 and 1 satisfies the upper threshold given in Eq. (7). Therefore, to generate a linear constraint on the BER, a linear expression for the sum of standard deviations is needed. One possible solution to this problem is to neglect the noise variances due to bit 0 and choose a slightly larger $Q$ factor than that corresponding to the target BER [15]. In this study, we use a different approach to guarantee that the resulting BER is lower than the threshold value. We search for the minimum value of a coefficient $K$, such that the following inequality is always satisfied:

$$
K\left(\sigma_{0}^{2}+\sigma_{1}^{2}\right) \geqslant\left(\sigma_{1}+\sigma_{0}\right)^{2} .
$$

The minimum value of $K$ is found using Eq. (10) and assuming the worst-case scenario on the network. Finally, the BER constraint is introduced as

$$
\begin{gathered}
M \times\left(1-x_{w d p}\right)+\sigma_{\text {lim }}^{2} \geqslant K\left(\sigma_{0}^{2}+\sigma_{1}^{2}\right), \\
\forall w \in W, d \in D, p \in P .
\end{gathered}
$$

The expression for $\sigma_{\text {lim }}^{2}$ is given in Eq. (6), and $\sigma_{0}^{2}$ $+\sigma_{1}^{2}$ is calculated from Eq. (10). The large constant $M$

TABLE I

Values of the Physical Model Parameters

\begin{tabular}{cll}
\hline$D_{p}$ & Fiber PMD Coefficient & $0.2 \mathrm{ps} / \sqrt{\mathrm{km}}$ \\
$\mathrm{B}$ & Channel data rate & $10 \mathrm{Gbps}$ \\
$G_{\text {in }}$ & Input EDFA gain & $22 \mathrm{~dB}$ \\
$G_{\text {out }}$ & Output EDFA gain & $16 \mathrm{~dB}$ \\
$X_{\text {sw }}$ & Switch cross-talk ratio & $-30 \mathrm{~dB}$ \\
$\xi_{\text {pol }}$ & Polarization mismatch factor & 0.5 \\
$R_{\lambda}$ & Photodetector responsivity & 1 \\
$B_{e}$ & Receiver electrical bandwidth & $B \times 0.7 \mathrm{GHz}$ \\
$B_{o}$ & Receiver optical bandwidth & $50 \mathrm{GHz}$ \\
$q$ & Charge of an electron & $1.16^{-19} \mathrm{C}$ \\
$\sqrt{n_{t h}}$ & RMS thermal current & $5.3^{-12} \mathrm{Amp} / \sqrt{\mathrm{Hz}}$ \\
\hline
\end{tabular}


is used for implementing the classical big-M linearization [29]. The use of $M$ in Eq. (12) guarantees that the inequality is satisfied when $x_{w d p}=0$, and the inequality becomes redundant when $x_{w d p}=1$. The smallest value of $M$, which is guaranteed to satisfy the inequality, is found by calculating the numerical value of the right-hand side expression in Eq. (10), when all the decision variables in the expression for $P_{X}(w, d, p)$ are taken as 1.

\section{REORDERED LIGHTPATH ESTABLISHMENT (ROLE) ALGORITHM}

The solution time of the ILP formulation introduced in Section IV increases exponentially with the problem size, and the extensive memory and running time requirements make the solution of the ILP formulation infeasible for medium- and large-size networks with large numbers of wavelengths. To obtain solutions for larger problem sizes, we propose a heuristic algorithm: reordered lightpath establishment (ROLE).

The objective of the ROLE algorithm is to route as many of the lightpath demands as possible without violating the BER constraints. ROLE basically routes the lightpath demands with different orders and chooses the best solution. It is implemented with various routing and wavelength assignment algorithms. It consists of three phases: RWA, rerouting, and reordering.

1) Routing and Wavelength Assignment: ROLE can employ various RWA algorithms. In this study, three routing and three wavelength assignment algorithms are implemented and their combinations are investigated. Together with these routing and wavelength assignment algorithms, two exhaustive RWA algorithms are also implemented to investigate all possible route-wavelength combinations for each lightpath demand.

In the routing process, the routes are chosen from the previously calculated shortest-path sets for each source-destination pair. To route a lightpath demand, the paths in the shortest-path set are searched for an available wavelength and the first route on which an available wavelength exists is chosen. An available wavelength is defined as a wavelength that is free on all the links along the chosen path and satisfies the BER constraints for the current lightpath and the already-established lightpaths. The search order for the routes is determined by the routing algorithm. The implemented routing algorithms in this study are the shortest path first (SPF), shortest widest path first (SWPF), and widest shortest path first (WSPF) algorithms. The width of a path is a dynamic metric and it is defined as the number of available wave- lengths along the path. The number of hops along the path is used as the path length.

For wavelength assignment, three algorithms are implemented: first fit with BER constraint (FFB), maximum BER (MB), and maximum-minimum BER (MMB). In the FFB algorithm, the wavelengths are numbered first. When assigning a wavelength to a lightpath demand, the smallest numbered available wavelength on the chosen path, which satisfies the BER constraints for that lightpath demand and the already-established lightpaths, is selected. In the MB and MMB algorithms, the resulting BERs for the current lightpath demand and the already-established lightpaths are calculated for all the available wavelengths on the chosen path. The MB algorithm assigns the wavelength that results in the lowest BER for the lightpath to establish and satisfies the BER constraints for that lightpath and the already-established lightpaths. The MMB algorithm assigns the wavelength that results in the lowest maximum BER among the lightpaths to establish and the alreadyestablished lightpaths, if the BER constraints are satisfied for these lightpaths. In all wavelength assignment algorithms, BER calculations are carried out using the $Q$-factor approach and decomposing the introduced $Q$-factor penalties as eye related and noise related, as explained in Section III and using Eqs. (1)-(6).

Besides these routing and wavelength assignment algorithms that can be run jointly, two exhaustive RWA algorithms are also implemented: exhaustive minimum BER (E-MB) and exhaustive minimummaximum BER (E-MMB). These algorithms are exhaustive in the sense that they do not stop the search when they find an available wavelength on a path, but search all path-wavelength combinations to find the best combination for the lightpath demand. E-MB chooses the path-wavelength combinations giving the minimum BER for the lightpath demand to establish. E-MMB chooses the path-wavelength combination that gives the minimum of the maximum BER among all the established lightpaths in the network.

2) Rerouting: In this phase, the objective is to improve the result of the RWA phase. After the RWA phase, the blocked lightpath demands are classified into two groups: wavelength-blocked demands and BER-blocked demands, i.e., lightpaths that are blocked due to capacity and BER constraints, respectively. The algorithm first tries to route the BERblocked demands. For each BER-blocked demand, the algorithm constructs a set of lightpaths that are routed on the same wavelength and share a common node with it. Then, it tries to reroute the lightpaths in this set. The aim of this procedure is to reduce the effect of the node cross-talk. The rerouting process continues until the BER-blocked lightpath can be routed 
or all the lightpaths in the set are tried. Then, the algorithm continues with the next BER-blocked lightpath.

The next step in this phase is to try routing the wavelength-blocked lightpath demands. For each wavelength-blocked lightpath demand, all the paths of the lightpath demand and all the wavelengths are searched to calculate the minimum number of reroutings needed to free an available wavelength along a path and the path and wavelength corresponding to this minimum. Then, the algorithm tries to reroute the lightpaths occupying the chosen wavelength on the links along the chosen path. If it is successful, the wavelength-blocked lightpath demand can be routed on that path and wavelength.

3) Reordering: At the start of the ROLE algorithm, the demands are sorted according to the length of their shortest paths. After the RWA and rerouting phases are run with the initial sorting order, the number of routed lightpath demands is recorded. The first lightpath demand that cannot be satisfied and is not marked is moved to the first place in the demand list and marked. Then, RWA and rerouting phases are run with the new sorting order. This reordering process is applied until there is no unsatisfied lightpath demand that is not marked, and the best result is recorded. Loops are avoided by marking the demands that are moved to the first place in the list and preventing them from being moved to the first place in another iteration.

The flow chart of the ROLE algorithm is given in Fig. 1.

Computational Complexity: The rerouting and reordering processes utilized in ROLE bring extra computational complexity to the employed RWA algorithm. The number of extra $Q$-factor calculations performed in the rerouting process is of the order of $O\left(|D|^{2}|W|^{2} k^{2} H^{2}\right)$, where $H$ denotes the average number of paths sharing at least one common node with a given path and $k$ is the number of considered shortest paths for each source-destination pair. The reordering process multiplies the total complexity of the RWA and rerouting phases by a factor of $|D|$.

\section{PERFormance Evaluation}

In this section, the performance of the ROLE algorithm is investigated. In the first part, the effect of the initial sorting of the lightpath demands is examined. When evaluating the solutions, the percentage of the routed lightpaths from the demand set is used as the performance metric. However, a solution may seem to show better performance by rejecting most of the lightpath demands between the nodes that are further away in the physical topology and only establishing

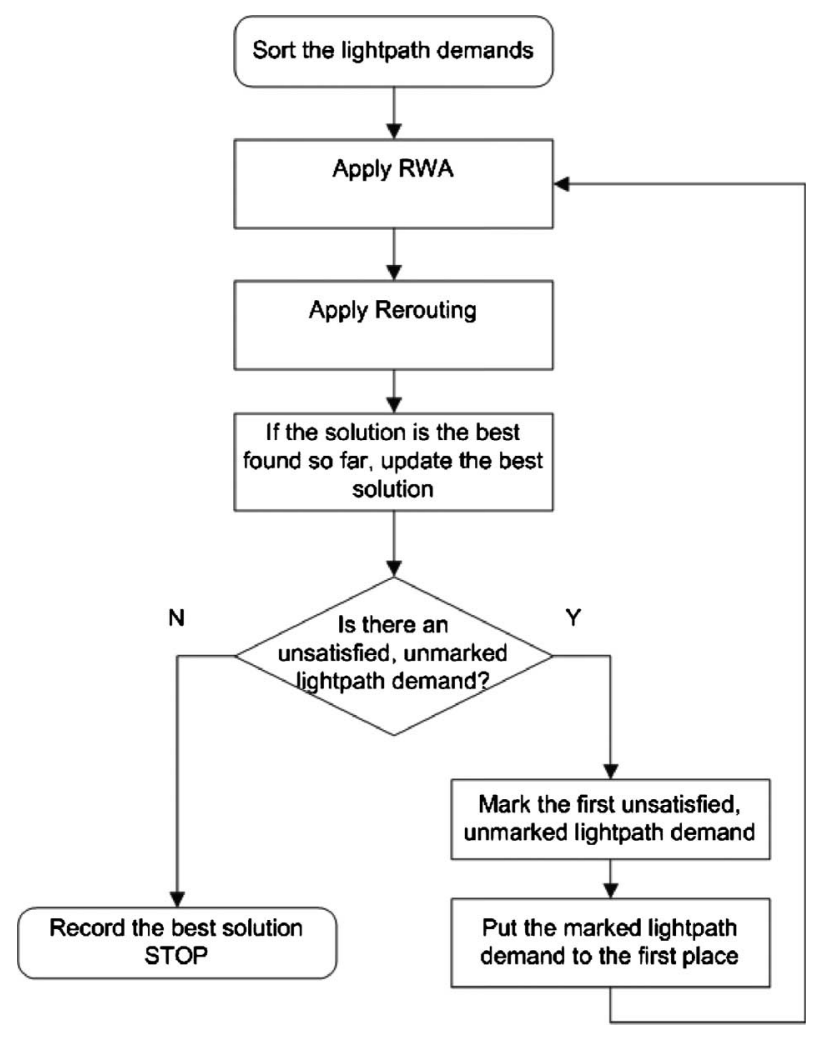

Fig. 1. Flow chart of the ROLE algorithm.

the lightpaths between closer nodes. This is not a desired situation since it violates fairness among the lightpath demands and it may also restrict the type of virtual topologies that can be established on top of the physical topology. Therefore, we also investigate the distribution of the blocking ratio as the path length changes to make a more comprehensive evaluation of the solutions.

The performance of ROLE is evaluated using different RWA algorithms. ROLE is run on four randomly generated physical topologies, each with 20 nodes and 25, 30, 35, and 40 bidirectional links (topologies 1-4, respectively). Simulations are run for three different numbers of wavelengths: $|W|=4,8$, and 16 . The lightpath demands, i.e., the logical topology, are generated randomly. The number of lightpath demands, $|D|$, in the demand set depends on $|W|$, and three sets of demands are generated for each value of $|D|$. For $|W|$ $=4,|D|=12,16,20,24,28,32,36,40$; for $|W|=8,|D|$ $=24,32,40,48,56,64,72,80 ;$ and for $|W|=16,|D|$ $=48,64,80,96,112,128,144,160$ are used. Ten shortest paths are calculated for each source-destination pair. The chosen threshold for the minimum value of the $Q$ factor is $6(15.5 \mathrm{~dB})$, which corresponds to a BER of about $10^{-9}$ without application of forward error correction (FEC) for a channel data rate of 10 Gbps.

In the next subsection, the performance of ROLE is compared with the ILP solutions for small-sized net- 


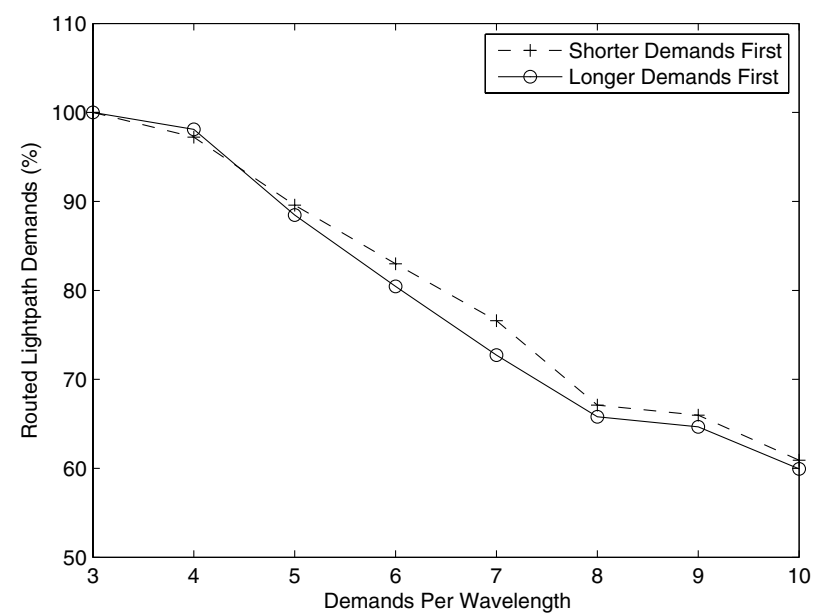

Fig. 2. Percentage of the routed demands by ROLE on topology 1 .

works. The ILP solutions are generated by solving the formulation introduced in Section 4. In the cases where an optimum solution could not be achieved, upper bounds and the best feasible solutions are reported.

ROLE is also evaluated on a realistic network topology based on the Deutsche Telekom (DTAG) network [30] for comparison with the POLIO-RWA algorithm [8] and two other algorithms that are derived from ROLE but do not apply rerouting and reordering. ROLE is shown to have a performance superior to the performances of the other algorithms. The performance improvements brought by the rerouting and reordering procedures are also shown. On the same physical topology, the effect of switch cross-talk, the most dominant physical layer impairment in the utilized model, and the percentage of wavelengthblocked and BER-blocked lightpath demands are also investigated.

\section{A. Effect of Initial Demand Sorting}

In this part, the effect of the initial sorting of the lightpath demands on the solutions produced by

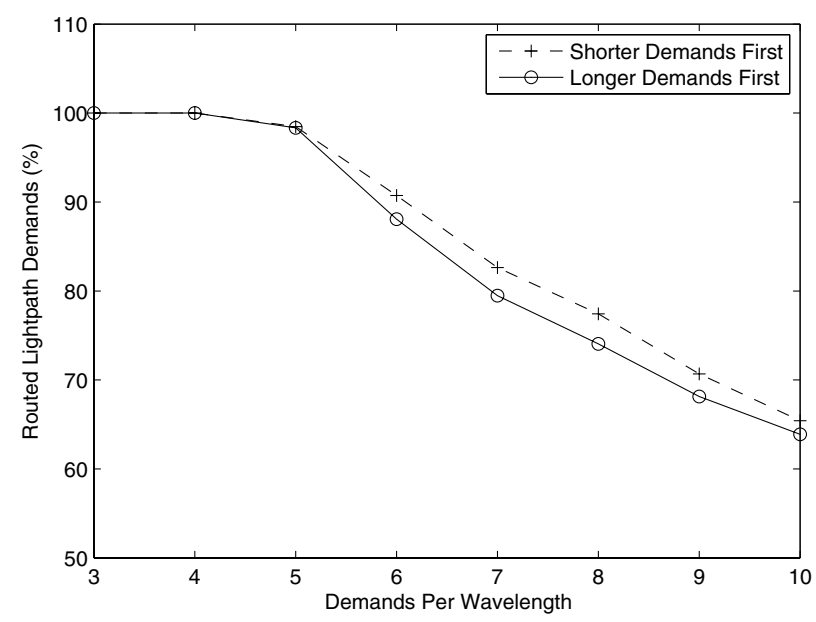

Fig. 3. Percentage of the routed demands by ROLE on topology 2 .

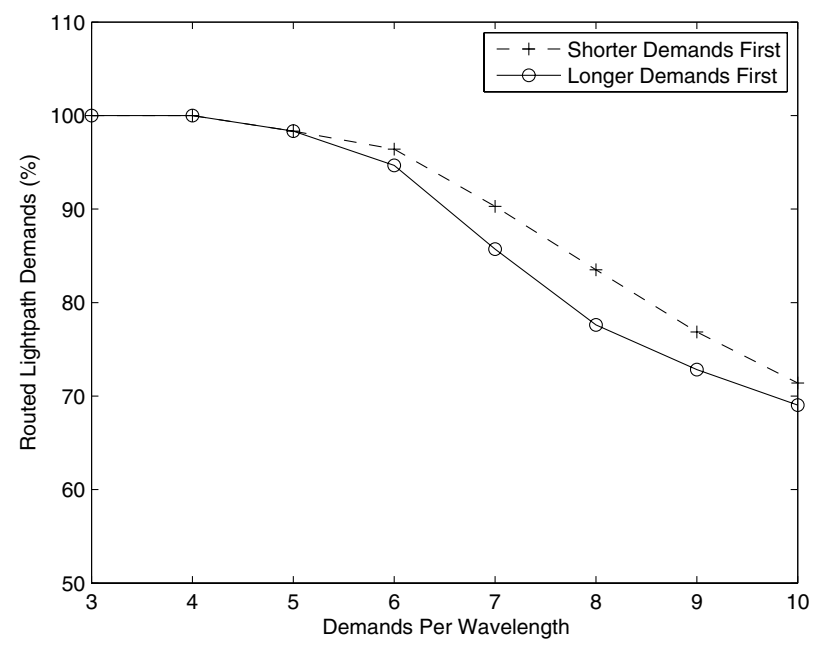

Fig. 4. Percentage of the routed demands by ROLE on topology 3.

ROLE is investigated. The length of a lightpath demand is defined as the shortest hop distance between the source and destination nodes of the demand on the physical topology. ROLE is run with two different initial sortings of the lightpath demands: shorter demands first (SDF) and longer demands first (LDF). The two approaches are compared in terms of the ratio of the lightpath demands that can be satisfied from the offered demand set, for different values of demands per wavelength $(|D| /|W|)$. The RWA algorithm employed by ROLE is SPF-FFB. The results are given in Figs. 2-5 for the 25-, 30-, 35-, and 40-link topologies referred to as topology 1 , topology 2 , topology 3 , and topology 4 , respectively.

The results show that, with SDF initial sorting, ROLE can satisfy a higher ratio of the offered lightpath demands and the maximum difference is approximately $5 \%$ of the offered lightpath demands. However, it is expected to have a tendency of denying the longer lightpath demands and thus causes unfair-

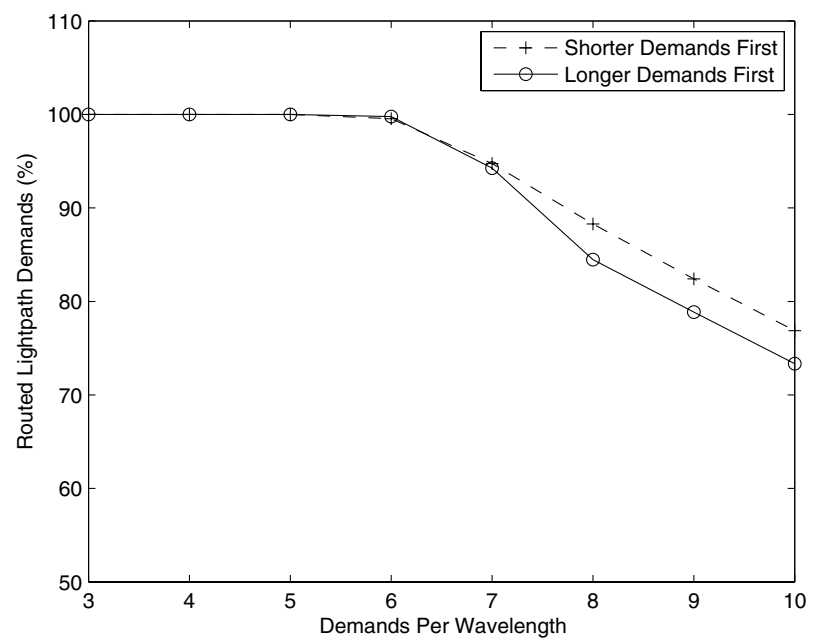

Fig. 5. Percentage of the routed demands by ROLE on topology 4. 


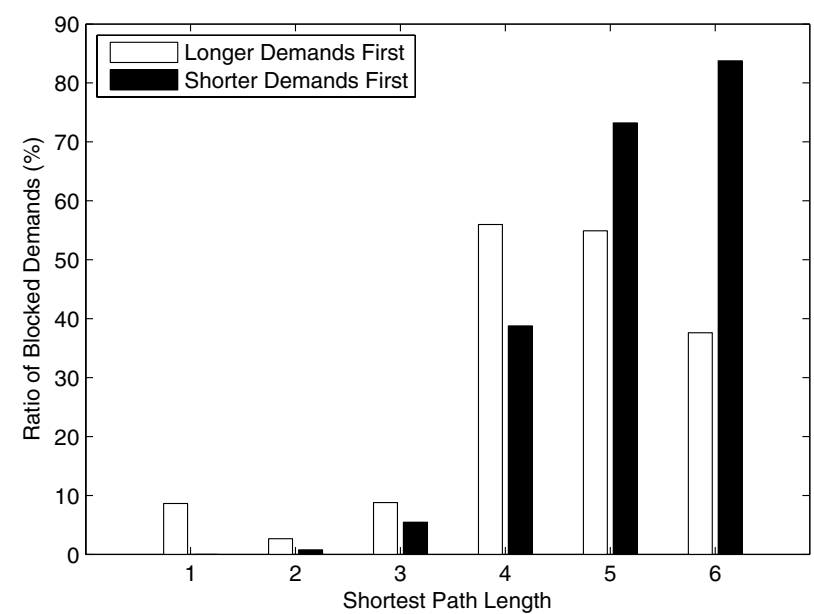

Fig. 6. Distribution of the blocked lightpath demands according to their lengths on topology 1.

ness among the offered demands and an important restriction on the type of virtual topologies that can be established. To examine this effect, we calculated the percentage of the blocked lightpath demands for each length. The results are presented in Figs. 6-9. The mean value and coefficient of variation (standard deviation/mean) for the lengths of the blocked lightpaths are given in Table II. As is observed from the results, the LDF initial sorting achieves a more fair distribution of the blocked lightpath demands in terms of demand lengths. The effect of initial demand sorting is investigated also on different topologies for larger values of $|W|$ up to $|W|=64$. The results are very similar to those reported here for $|W|=4,8,16$; therefore they are not included in the paper.

\section{B. Performance With Different RWA Algorithms}

The performance of ROLE with different RWA approaches described in the previous subsection are evaluated in terms of the ratio of the routed lightpath

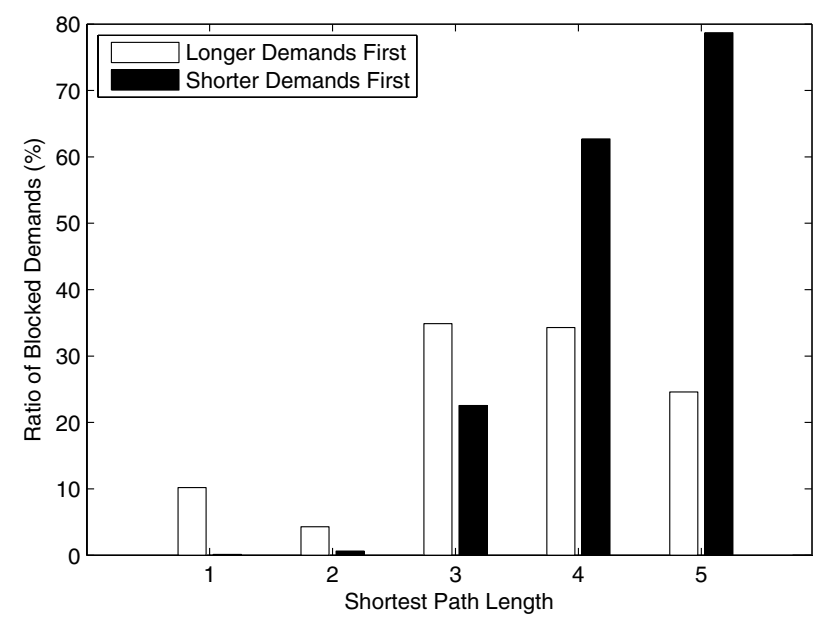

Fig. 7. Distribution of the blocked lightpath demands according to their lengths on topology 2.

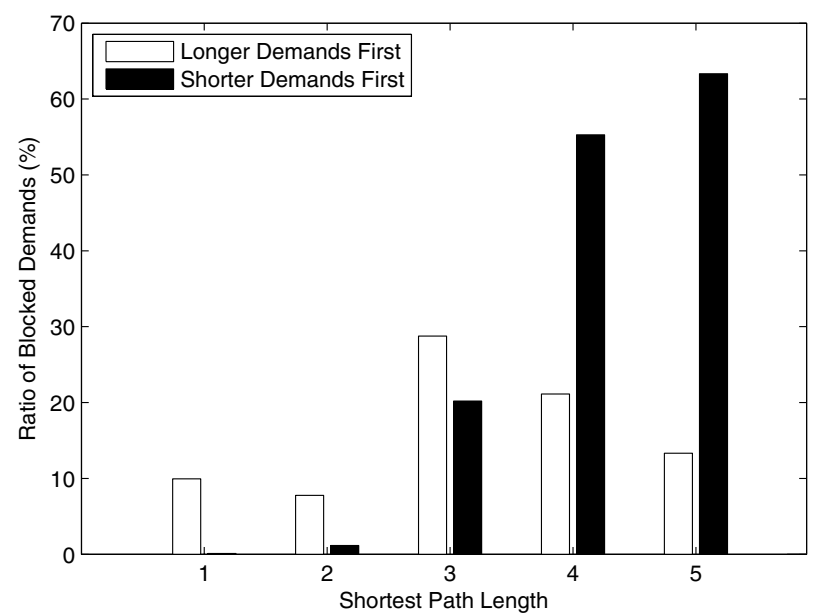

Fig. 8. Distribution of the blocked lightpath demands according to their lengths on topology 3.

demands. Each of the three routing algorithms (SPF, WSPF, and SWPF) are run with the three wavelength assignment algorithms (FFB, MB, and MMB), and two exhaustive RWA algorithms (E-MB and E-MMB) are also implemented. The results are shown in Figs. 10-13 for topologies 1-4. In the results, each routing algorithm is paired with the wavelength assignment algorithm it performs best, and the best of the two exhaustive algorithms is chosen. The initial demand

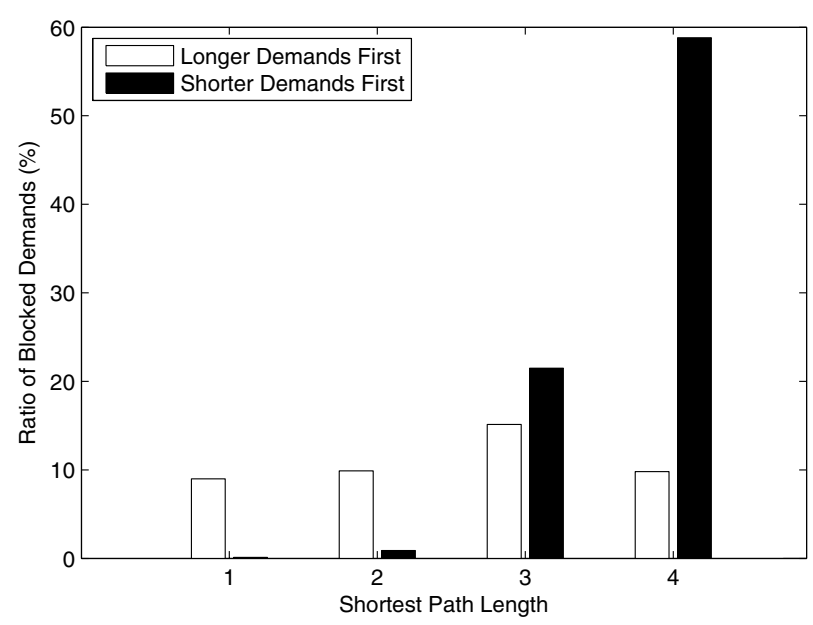

Fig. 9. Distribution of the blocked lightpath demands according to their lengths on topology 4.

TABLE II

Statistics ON THE LENGTHS OF THE BLOCKed LightPathS FOR SDF AND LDF DEMAND SORTING ON TOPOLOGIES 1-4

\begin{tabular}{cccccc}
\hline & \multicolumn{2}{c}{ Mean Length } & & \multicolumn{2}{c}{ Coe. Var. } \\
\cline { 2 - 3 } \cline { 5 - 6 } Topology & SDF & LDF & & SDF & LDF \\
\hline 1 & 4.65 & 4.20 & & 0.16 & 0.24 \\
2 & 3.63 & 3.05 & & 0.17 & 0.28 \\
3 & 3.54 & 2.76 & & 0.19 & 0.32 \\
4 & 3.17 & 2.34 & & 0.15 & 0.33 \\
\hline
\end{tabular}




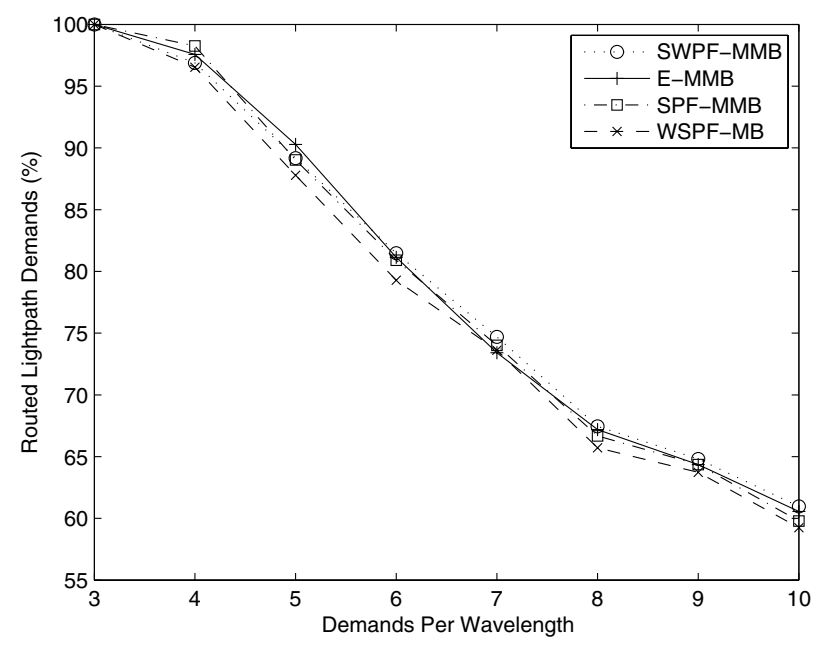

Fig. 10. Percentage of the routed demands by ROLE with different RWA algorithms on topology 1 .

sorting is LDF. The results averaged on all values of demands per wavelength and on all topologies are presented in Table III.

According to the results, SWPF performs the best among the routing algorithms, when incorporated in ROLE. SPF has close performance, and WSPF performs considerably worse than the other two routing algorithms. Among the wavelength assignment algorithms, the performances of MB and MMB are similar and better than that of the FFB algorithm. The performances of the exhaustive RWA algorithms are also among the best together with the SPF and SWPF algorithms employing MB and MMB for wavelength assignment. The best performance is achieved by the SWPF-MB combination.

We investigated the reason for the close performance of the $\mathrm{MB}$ and MMB wavelength assignment algorithms. When a lightpath demand is blocked because of high BER, there are two possible cases that

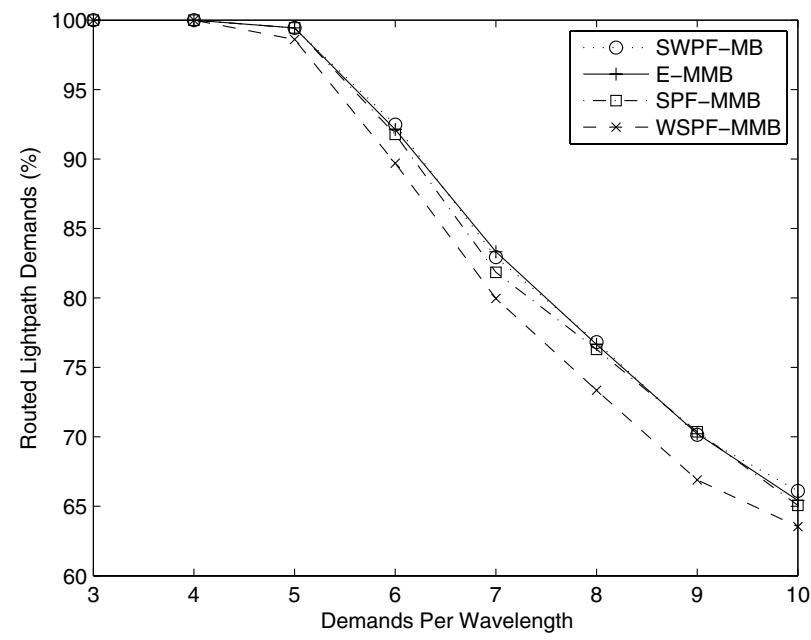

Fig. 11. Percentage of the routed demands by ROLE with different RWA algorithms on topology 2.

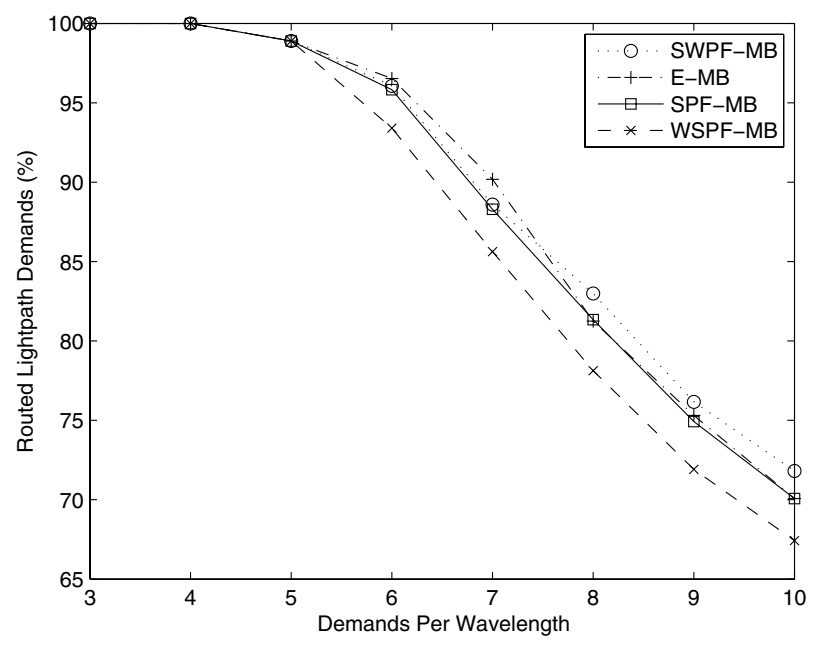

Fig. 12. Percentage of the routed demands by ROLE with different RWA algorithms on topology 3 .

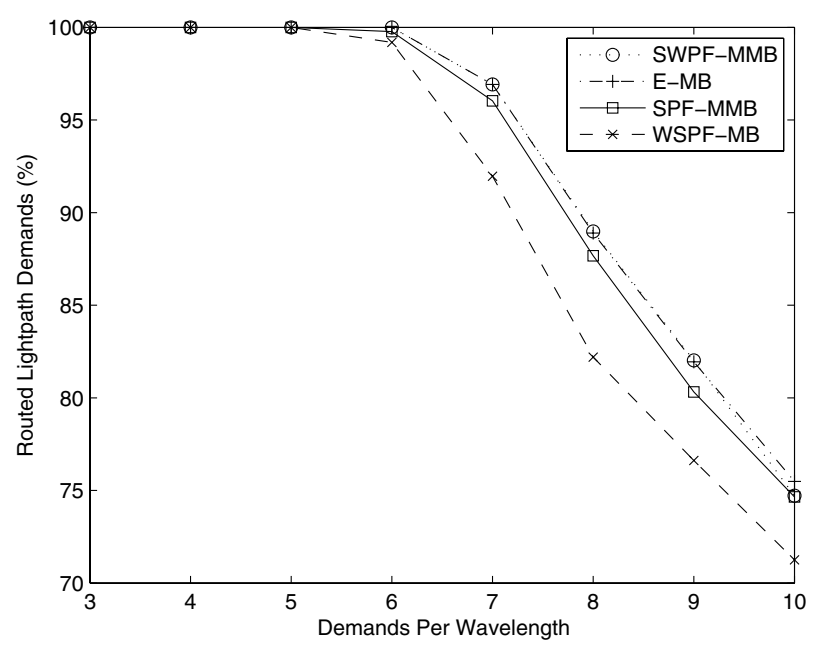

Fig. 13. Percentage of the routed demands by ROLE with different RWA algorithms on topology 4 .

TABLE III

Percentage of the Established Lightpath Demands by ROLE With Different RWA Algorithms Averaged oN TOPOLOGIES 1-4

\begin{tabular}{ccc}
\hline \multicolumn{2}{c}{ RWA Algorithm } & Routed \% \\
\hline SPF & FFB & 85.34 \\
& MB & 86.31 \\
& MMB & 86.37 \\
SWPF & FFB & 85.95 \\
& MB & 86.81 \\
\multirow{2}{*}{ WSPF } & MMB & 86.79 \\
& FFB & 83.16 \\
& MB & 84.82 \\
Exhaustive & MMB & 84.78 \\
& MB & 86.69 \\
& MMB & 86.63 \\
\hline
\end{tabular}


may have occurred. The first possible case is that the current lightpath demand to establish exceeds the BER threshold and thus has the highest BER in the network, since already-established lightpaths satisfy the BER constraints. The second case is that the BER of the current lightpath demand remains below the acceptable threshold, but it causes the BER of an already-established lightpath to increase and exceed the threshold. In both cases, the lightpath demand to be established is blocked. We computed the average percentage of blocked lightpath requests that fall into the first category, where the average is taken over all simulation events using topologies $1-4$. This percentage is about $68 \%$ for both the SPF-MB and SPF-MMB algorithms, i.e., in $68 \%$ of the blockings due to high BER, the current lightpath demand to establish has the maximum BER among all the lightpaths in the network and the blocking occurs due to the BER of the current lightpath demand. It can be deduced that, in such cases applying the MinMax approach would also result in the blocking of the lightpath demand since the blocking is not due to the BER of other lightpaths.

To further investigate the performance difference between the MB and MMB algorithms, we calculated the maximum BER among the lightpaths in the established virtual topologies when the SPF-MB and SPFMMB RWA algorithms are used. The results that are averaged over all demand sets and wavelengths are presented in Table IV. As is seen from the results, except for the simulations run on topology 1 , which has the smallest number of physical links and therefore the lowest amount of physical layer resources, the average value of the maximum BER in the virtual topologies established by the MMB algorithm is lower. Although SPF-MMB slightly outperforms SPF-MB in terms of average maximum BER, the number of lightpaths that do not satisfy the maximum BER threshold is very close for the two algorithms. Therefore, their performances are similar in terms of the amount of established lightpaths.

TABLE IV

Average Values of the Maximum BERs Among the LightPaths IN THE Established ViRTUAL TOPOLOGIES ON TOPOLOGIES 1-4

\begin{tabular}{ccc}
\hline & \multicolumn{2}{c}{ RWA Algorithm } \\
\cline { 2 - 3 } Topology & SPF-MB & SPF-MMB \\
\hline 1 & $3.9 \times 10^{-10}$ & $3.94 \times 10^{-10}$ \\
2 & $2.96 \times 10^{-10}$ & $2.72 \times 10^{-10}$ \\
3 & $2.71 \times 10^{-10}$ & $2.57 \times 10^{-10}$ \\
4 & $2.26 \times 10^{-10}$ & $2.18 \times 10^{-10}$ \\
\hline
\end{tabular}

\section{Comparison With ILP Solutions}

In this part, the solutions generated by ROLE and the solutions of the ILP formulation presented in Section IV are compared on smaller-size networks, for which the ILP formulation can be solved to optimality or near optimality. Three randomly generated physical topologies, each with 10 nodes, are used for this purpose: topology 5 , topology 6 , and topology 7 , with 12,16 , and 20 links, respectively. Simulations are run for $|W|=4,8,12$. For $|W|=4,|D|=10,15,20,25$; for $|W|$ $=8, \quad|D|=20,30,40,50 ; \quad$ and for $|W|=12, \quad|D|$ $=30,45,60,75$.

ROLE is run with the E-MMB RWA algorithm with the SDF and LDF demand sorting orders. The ILP formulation is solved using the ILOG CPLEX 8.1 optimization problem solver, applying the branch and cut method. The solver is run with an upper limit on the spent CPU time of $10,000 \mathrm{~s}$. On the other hand, the longest run time for ROLE was $8 \mathrm{~s}$ in the studied cases. For the cases in which the problem could not be solved to optimality within the run-time limit, the best solution produced by branch and cut is recorded as the ILP solution. An upper bound is also produced by the branch and cut algorithm, by taking the best noninteger solution in the unexplored region.

The number of the routed lightpath demands is given in Tables V-VII for topologies 5, 6, and 7, respectively. As can be seen from the results, the ILP formulation could be solved to optimality for smaller values of $|W|$ and $|D|$, but as the problem size grows, it cannot be solved to optimality within the applied CPU time constraint.

The ILP solutions on topologies 5 and 6 are slightly better than the solutions produced by the heuristic ROLE with SDF initial demand sorting. As the prob-

\section{TABLE V}

Number OF ROUTED DEMANDS WITH THE ILP AND Heuristic SOlutions AND UPPER Bounds FOR TOPOLOGY $5^{a}$

\begin{tabular}{cccccc}
\hline$|W|$ & $|D|$ & U.B. & ILP & $\begin{array}{c}\text { ROLE } \\
\text { SDF }\end{array}$ & $\begin{array}{c}\text { ROLE } \\
\text { LDF }\end{array}$ \\
\hline \multirow{2}{*}{4} & 10 & 10 & $* 10$ & ${ }^{*} 10$ & $* 10$ \\
& 15 & 14 & $* 14$ & $* 14$ & ${ }^{*} 14$ \\
& 20 & 17 & $* 17$ & 16 & 16 \\
& 25 & 17 & $* 17$ & $* 17$ & 16 \\
& 20 & 20 & $* 20$ & $* 20$ & $* 20$ \\
& 30 & 29 & 28 & 28 & 28 \\
& 40 & 35 & 31 & 30 & 29 \\
& 50 & 37 & 34 & 33 & 33 \\
& 30 & 30 & $* 30$ & $* 30$ & $* 30$ \\
& 45 & 44 & $* 44$ & 42 & 42 \\
& 60 & 53 & 50 & 48 & 48 \\
& 75 & 52 & 50 & 47 & 44 \\
\hline \multirow{2}{*}{ Total } & 420 & 358 & 345 & 335 & 330 \\
\hline
\end{tabular}

$a_{*}$ denotes the optimum solution 
TABLE VI

Number OF ROUTED DEMANDS WITH THE ILP AND Heuristic Solutions AND UpPer Bounds For TOPOLOGy $6^{a}$

\begin{tabular}{cccccc}
\hline$|W|$ & $|D|$ & U.B. & ILP & $\begin{array}{c}\text { ROLE } \\
\text { SDF }\end{array}$ & $\begin{array}{c}\text { ROLE } \\
\text { LDF }\end{array}$ \\
\hline \multirow{2}{*}{4} & 10 & 10 & ${ }^{*} 10$ & ${ }^{*} 10$ & ${ }^{*} 10$ \\
& 15 & 13 & ${ }^{*} 13$ & ${ }^{*} 13$ & ${ }^{*} 13$ \\
& 20 & 18 & 17 & 16 & 15 \\
& 25 & 22 & 18 & 17 & 16 \\
8 & 20 & 20 & ${ }^{*} 20$ & ${ }^{*} 20$ & ${ }^{*} 20$ \\
& 30 & 30 & ${ }^{*} 30$ & ${ }^{*} 30$ & ${ }^{*} 30$ \\
& 40 & 39 & 33 & 33 & 33 \\
12 & 50 & 43 & 37 & 36 & 35 \\
& 30 & 30 & ${ }^{*} 30$ & ${ }^{*} 30$ & ${ }^{*} 30$ \\
& 45 & 45 & ${ }^{*} 45$ & ${ }^{*} 45$ & ${ }^{*} 45$ \\
& 60 & 59 & 51 & 51 & 50 \\
Total & 75 & 66 & 54 & 54 & 54 \\
\hline$*$
\end{tabular}

$a_{*}$ denotes the optimum solution

lem size grows with the number of links on the physical topology, ROLE with SDF starts to outperform the ILP solution obtained within run-time limits, as in the case for topology 7 .

The distribution of the blocked lightpath demands according to the shortest path lengths is presented in Figs. 14-16 for topologies 5, 6, and 7, respectively. The mean lengths and coefficients of variation (standard deviation/mean length) for the blocked lightpaths are given in Table VIII. As the results show, longer lightpath demands have a higher probability of rejection also in the ILP solutions. Applying LDF initial demand sorting with ROLE, it is possible to achieve a more fair distribution and lower probability of rejection for longer lightpath demands in exchange for a small reduction in the number of routed demands.

TABLE VII

Number OF ROUTED DEMANDS WITH THE ILP AND Heuristic Solutions AND UPPER Bounds FOR TOPOLOGY $7^{a}$

\begin{tabular}{cccccc}
\hline & & & & ROLE \\
& $|W|$ & & U.B. & ILP & $\begin{array}{c}\text { ROLE } \\
\text { LDF }\end{array}$ \\
\hline \multirow{2}{*}{4} & 10 & 10 & ${ }^{*} 10$ & ${ }^{*} 10$ & ${ }^{*} 10$ \\
& 15 & 13 & ${ }^{*} 13$ & ${ }^{*} 13$ & ${ }^{*} 13$ \\
& 20 & 20 & 19 & 18 & 19 \\
& 25 & 24 & 21 & 21 & 18 \\
8 & 20 & 20 & ${ }^{*} 20$ & ${ }^{*} 20$ & ${ }^{*} 20$ \\
& 30 & 30 & ${ }^{*} 30$ & ${ }^{*} 30$ & ${ }^{*} 30$ \\
& 40 & 40 & 39 & 38 & 38 \\
& 50 & 47 & 40 & 41 & 39 \\
12 & 30 & 30 & ${ }^{*} 30$ & ${ }^{*} 30$ & ${ }^{*} 30$ \\
& 45 & 45 & ${ }^{*} 45$ & ${ }^{*} 45$ & ${ }^{*} 45$ \\
& 60 & 60 & 57 & ${ }^{*} 60$ & ${ }^{*} 60$ \\
\multirow{2}{*}{ Total } & 75 & 75 & 61 & 62 & 61 \\
& 420 & 414 & 385 & 388 & 383 \\
\hline
\end{tabular}

\footnotetext{
$a_{*}$ denotes the optimum solution
}

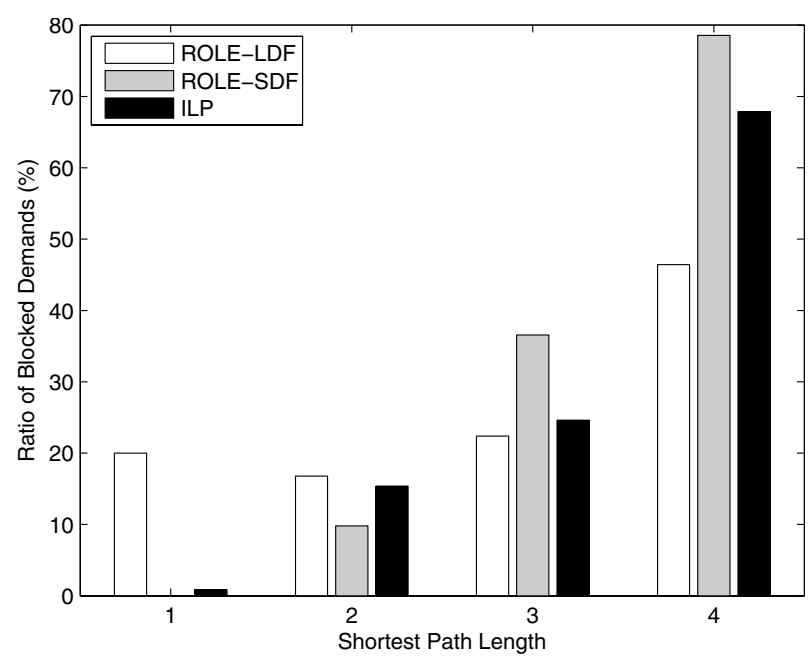

Fig. 14. Distribution of the blocked lightpath demands according to their lengths on topology 5 .

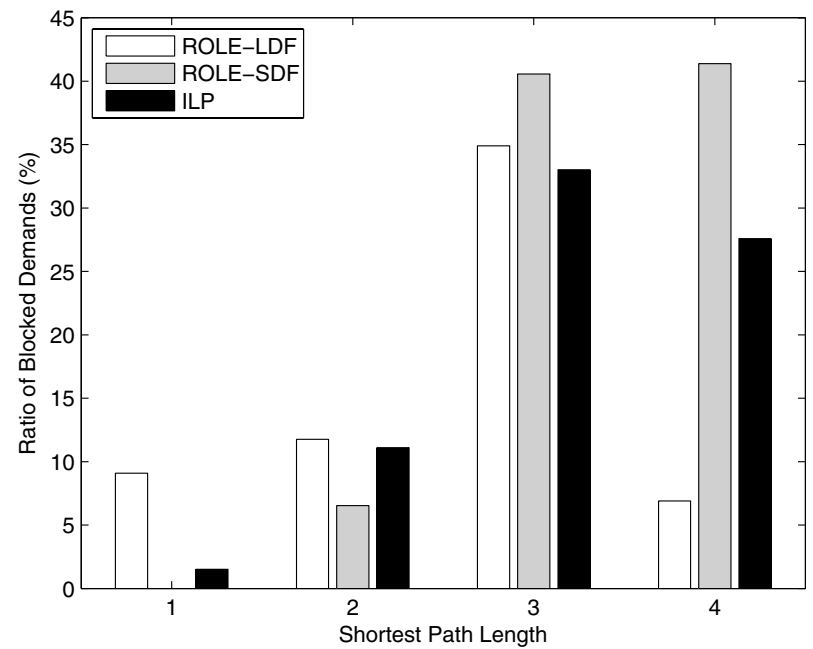

Fig. 15. Distribution of the blocked lightpath demands according to their lengths on topology 6.

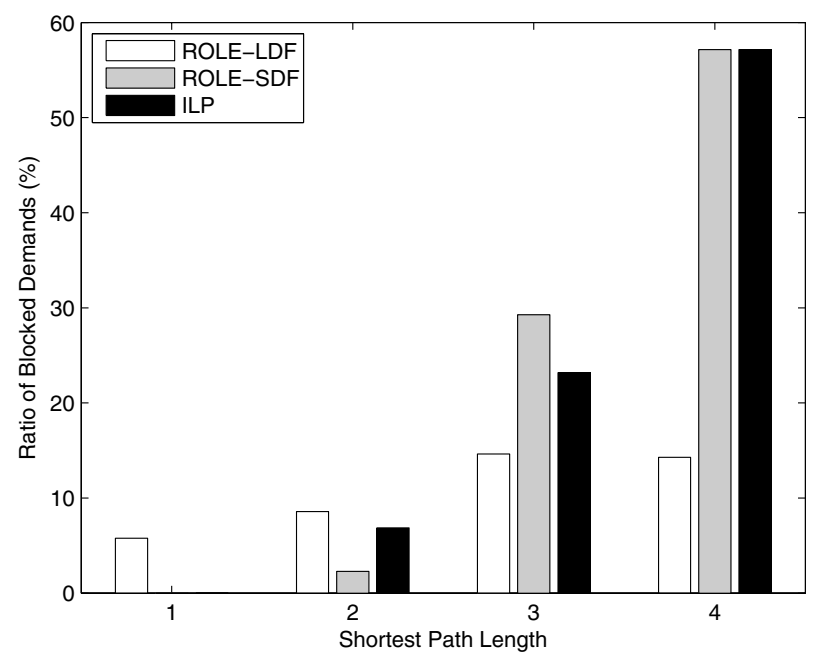

Fig. 16. Distribution of the blocked lightpath demands according to their lengths on topology 7 . 
TABLE VIII

Statistics ON THE Lengths OF THE Blocked LightPaths FOR THE ILP SOLUTION AND ROLE WITH SDF AND LDF

Demand Sorting on Topologies 5-7

\begin{tabular}{ccccccccc}
\hline & \multicolumn{3}{c}{ Mean Length } & & \multicolumn{3}{c}{ Coe. Var. } \\
\cline { 2 - 4 } \cline { 7 - 8 } Topology & ILP & $\begin{array}{c}\text { ROLE } \\
\text { SDF }\end{array}$ & $\begin{array}{c}\text { ROLE } \\
\text { LDF }\end{array}$ & & ILP & $\begin{array}{c}\text { ROLE } \\
\text { SDF }\end{array}$ & $\begin{array}{c}\text { ROLE } \\
\text { LDF }\end{array}$ \\
\hline 5 & 2.93 & 3.09 & 2.37 & & 0.26 & 0.21 & 0.43 \\
6 & 2.79 & 3.03 & 2.42 & & 0.25 & 0.19 & 0.33 \\
7 & 2.77 & 3.00 & 2.14 & & 0.23 & 0.17 & 0.38 \\
\hline
\end{tabular}

\section{Comparison With Other Heuristics}

In this subsection, we compare the performance of ROLE with other heuristic algorithms. One of them is the POLIO-RWA algorithm proposed in [8]. In this algorithm, the lightpath demands are sorted according to the lengths of the shortest paths between the source and destination nodes in an increasing manner, as in the SDF sorting, and served in this preprocessed order. For each demand, the $k$ shortest and $\delta$ distinct paths are calculated dynamically on a layered wavelength graph where only the links with available wavelengths are considered. RWA is performed in a way to minimize the maximum BER among all established lightpaths and if no route-wavelength combination satisfying the BER constraints is found, the lightpath demand is blocked. The algorithm is run with the parameters $k=10$ and $\delta=2$

Two other heuristic algorithms, lightpath establishment without rerouting (LERR) and lightpath establishment without reordering (LERO), are also implemented to demonstrate the improvement brought by the rerouting and reordering mechanisms utilized in ROLE. In these algorithms, the lightpath demands are sorted and served in the SDF order. RWA is performed using the SWPF-MB algorithm. Both algorithms perform RWA once and do not apply reordering. LERR stops after the RWA process while LERO also performs rerouting as in the ROLE algorithm. Both of these heuristics and ROLE are run with $k$ $=10$, i.e., 10 shortest paths for each sourcedestination pair.

The simulations are carried out on the reference Deutsche Telekom network [30] with 14 nodes and 23 bidirectional links with $|W|=16$. The percentage of routed lightpath demands is calculated for different values of $|D|$ between 24 and 132, and 10 demand sets are generated for each value.

The percentages of established lightpaths for the four algorithms are given in Fig. 17. POLIO-RWA and LERR have similar performances in terms of number of established lightpaths. The difference between the performance of LERR and LERO shows the effect of

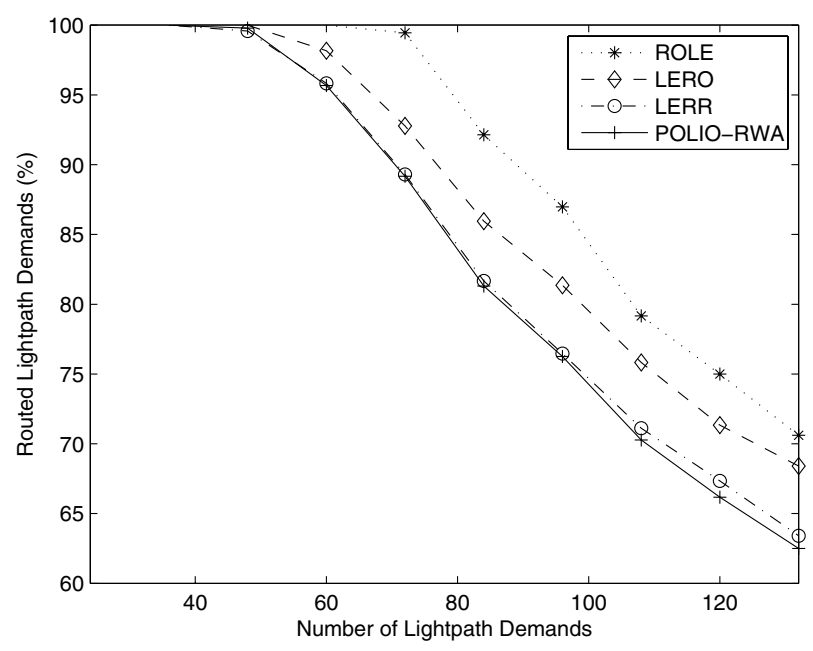

Fig. 17. Percentage of the routed demands by the heuristic algorithms on the DTAG topology.

the rerouting process. According to the results, applying rerouting increases the performance of LERR up to $7.9 \%$. ROLE clearly outperforms the other three algorithms in terms of number of routed lightpath demands. ROLE has a performance up to $14 \%$ higher than POLIO-RWA and LERR and up to $7.2 \%$ higher than LERO. The results verify that the rerouting and reordering mechanisms utilized in ROLE provide significant performance improvement in terms of routed lightpath demands, and ROLE is superior to the compared heuristics.

\section{E. Influence of Switch Cross-Talk Ratio and Number of Wavelengths}

With the choice of parameters used in this study, the most dominant physical layer impairment source for transmission errors turned out to be the node cross-talk occurring in the optical switches. In this subsection, we investigate the effect of the switch cross-talk ratio $\left(X_{s w}\right)$ on the amount of routed lightpaths and the amount of lightpath demands that are blocked due to high BER. The behavior of ROLE and the effect of $X_{s w}$ in the large $|W|$ regime are also investigated in order to evaluate the effect of the number of wavelengths.

Simulations are carried out on the DTAG network topology for $|W|=16$ and $|W|=128$. ROLE is run for different values of $X_{s w}$. The initial demand sorting order is SDF. For $|W|=16$, ROLE is run with the SWPF-FFB and E-MMB RWA algorithms, and for $|W|=128$, it is run with the SWPF-FFB algorithm. For $|W|=128$, the number of lightpath demands are increased proportionally with $|W|$, so that the number of lightpath demands per wavelength $(|W| /|D|)$ remains the same as in the case for $|W|=16$. The percentage of routed demands for various numbers of lightpath demands are shown in Figs. 18 and 19 for $|W|=16$ and $|W|=128$, re- 


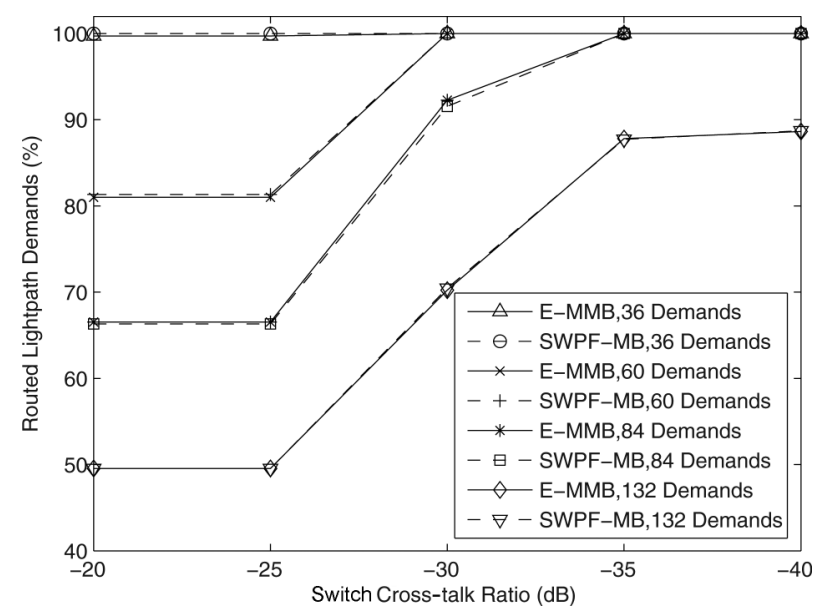

Fig. 18. Routed demand percentage versus switch cross-talk ratio for various numbers of lightpath demands for $|W|=16$.

spectively. As seen in Fig. 18, the E-MMB and SWPFFFB algorithms show very close performance when incorporated with ROLE, which is consistent with the results of Subsection VI.B. Therefore, ROLE is not run with the E-MMB algorithm, which has a higher complexity, for $|W|=128$. The change in the number of routed demands with $X_{s w}$ is similar for both $|W|=16$ and $|W|=128$, with the difference that the number of routed lightpaths per wavelength is higher to some extent, for $|W|=128$, due to more efficient statistical multiplexing as the number of wavelengths increases.

The results show that, for a switch cross-talk value larger than $-25 \mathrm{~dB}$, the percentage of routed demands does not decrease further as the cross-talk increases. After this value, the switch cross-talk ratio has the maximum effect and in the generated solutions no two lightpaths having a common node in their paths are assigned the same wavelength. For cross-talk values of $-35 \mathrm{~dB}$ and smaller, the switch cross-talk ratio has little effect on the percentage of blocked lightpath demands.

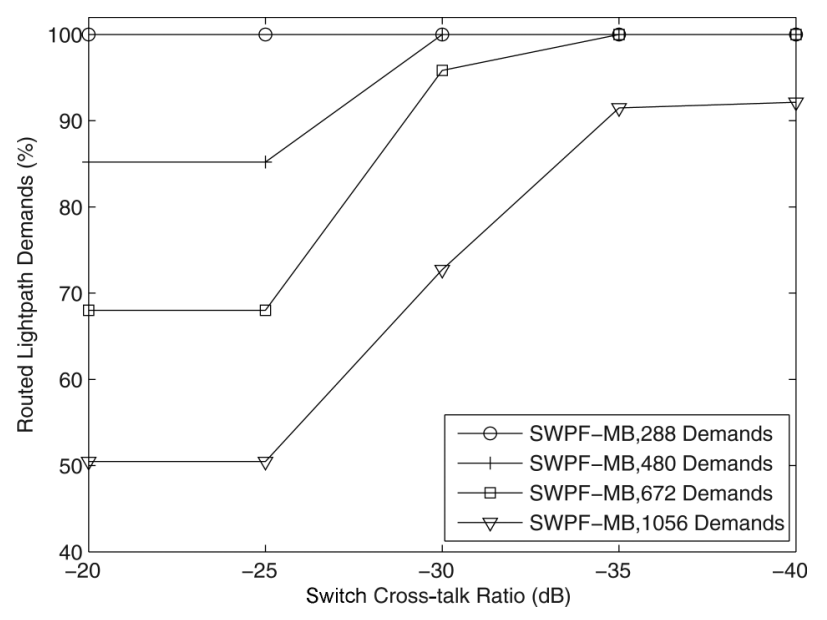

Fig. 19. Routed demand percentage versus switch cross-talk ratio for various numbers of lightpath demands for $|W|=128$.
To find out the major factor of lightpath demand blockings, we investigate the ratio of wavelengthblocked and BER-blocked lightpath demands, i.e., lightpath demands that are blocked due to capacity and BER constraints, respectively. These ratios are shown in Fig. 20 for $|W|=16$ and $|W|=128$ for various values of $X_{s w}$. The number of offered lightpath demands is 132 for $|W|=16$ and 1056 for $|W|=128$. As can be seen from the results, BER blocking is the dominant blocking type for larger values of $X_{s w}$, and for $-35 \mathrm{~dB}$ and smaller values, most of the blockings are due to insufficient capacity.

Figure 21 shows the change of the wavelengthblocked and BER-blocked lightpath demands with various numbers of lightpath demands for $X_{s w}$ $=-30 \mathrm{~dB}$. The capacity is sufficient to route all the offered lightpath demands when the number of offered demands does not exceed 96 for $|W|=16$ and 864 for $|W|=128$ and all blockings are due to physical impairments. After these values, the increase in the blocked lightpath demands is due to insufficient capacity, and as the number of offered lightpath demands increases, wavelength blockings also increase and become more dominant.

As the number of wavelengths and the number of offered lightpath demands increase proportionally, the percentage of routed lightpaths also increases, as can be seen from Figs. 18-21. Figures 20 and 21 suggest that the reason for this increase is the amount of lightpath demands that are blocked due to insufficient capacity, rather than high BER. That is an expected result because, for larger numbers of lightpath demands, the demands are distributed more fairly and a smaller number of bottlenecks are created in the network.

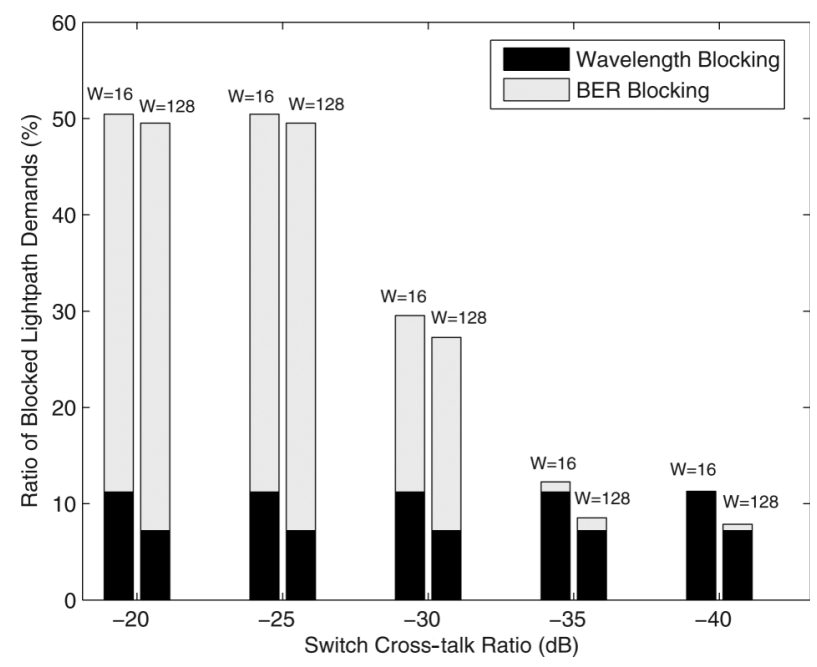

Fig. 20. Ratio of wavelength-blocked and BER-blocked lightpath demands versus switch cross-talk ratio. 


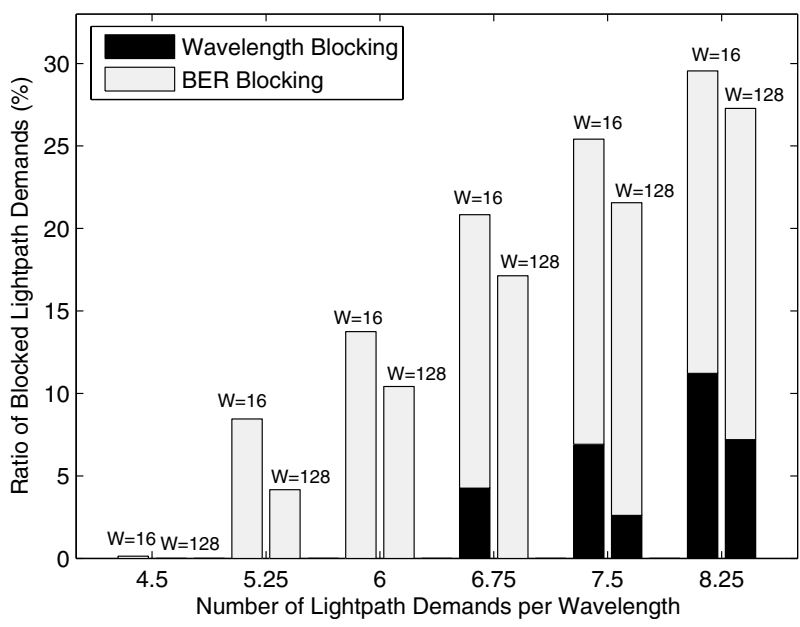

Fig. 21. Ratio of wavelength-blocked and BER-blocked lightpath demands versus number of offered lightpath demands per wavelength.

\section{CONCLUSIONS}

In this paper, we have investigated the lightpath establishment problem in the presence of physical layer impairments. This problem arises in the optical layer in MTE application scenarios. We proposed an efficient heuristic solution for the lightpath establishment problem: ROLE. We compared ROLE with POLIO-RWA, a recently proposed heuristic algorithm for the same problem that is shown to be superior to previously proposed heuristics [8]. ROLE is shown to have a performance up to $14 \%$ higher than POLIORWA in terms of number of established lightpath demands. ROLE can be run with different RWA algorithms. The most commonly used RWA algorithms are implemented and incorporated with ROLE, and their performances are evaluated.

An ILP formulation for the lightpath establishment problem is also developed. In the developed formulation, hard constraints are imposed on BERs, taking the aggregated effect of physical impairments into account. Since the considered physical layer impairments include node cross-talk, the chosen route and wavelength for a lightpath may affect the BER of other lightpaths. This fact makes the size of the ILP formulation excessively large. Therefore, the ILP formulation is used for evaluating the performance of ROLE on smaller-size networks. ROLE is shown to have a performance close to the optimum solutions for all the problem sets, for which the optimum solution can be obtained.

In the solutions obtained by solving the ILP formulation, longer lightpath demands, i.e., lightpath demands with longer shortest paths, have a higher blocking rate compared with the shorter lightpath demands. Changing the initial demand sorting order, ROLE can achieve a more fair distribution of blocked lightpath demands according to their lengths, at the expense of a slight reduction in the number of routed lightpath demands. This feature may bring a significant advantage since it allows more flexibility in designing the virtual topology at the upper layer.

We also investigated the major factor for lightpath demand blockings. Among the considered physical impairments, the most dominant one was the intrachannel node cross-talk. Therefore, we calculated the amount of lightpath demands blocked due to BER constraints and due to capacity constraints for varying values of the switch cross-talk ratio $\left(X_{s w}\right)$. The results show that for values of $X_{s w}$ smaller than or equal to $-35 \mathrm{~dB}$, most of the lightpath blockings are due to capacity constraints. For $X_{s w}$ values larger than $-30 \mathrm{~dB}$, physical impairments are the dominant factor for blockings. At $-25 \mathrm{~dB}, X_{s w}$ reaches its maximum effect, beyond this value no two lightpaths having a common node on their paths are routed on the same wavelength. The ratio of lightpath demands blocked due to BER constraints and the effect of $X_{s w}$ are similar also for large values of $|W|$. However, for larger numbers of wavelengths and lightpath demands, the ratio of blocked lightpaths due to capacity constraints decreases since the distribution of the lightpath demands becomes more balanced with the increasing amount of traffic.

\section{ACKNOWLEDGMENTS}

The work described in this paper was carried out with the support of the BONE project ("Building the Future Optical Network in Europe"), a Network of Excellence funded by the European Commission through the 7th ICT-Framework Programme, and by the Scientific and Technological Research Council of Turkey (TUBITAK) under the project 104E047. Namik Sengezer is supported in part by a graduate scholarship from TUBITAK.

\section{REFERENCES}

[1] R. Sabella, H. Zang, and E. Labonatory, "Guest editorial: Traffic engineering in optical networks," IEEE Network, vol. 17, no. 2, pp. 6-7, 2003.

[2] X. Xiao, A. Hannan, B. Bailey, and L. Ni, "Traffic engineering with MPLS in the Internet," IEEE Network, vol. 14, no. 2, pp. 28-33, 2000.

[3] D. Awduche, J. Malcolm, J. Agogbua, M. O’Dell, and J. McManus, "Requirements for traffic engineering over MPLS," IETF RFC 2702, 1999.

[4] D. Awduche, L. Berger, D. Gan, T. Li, V. Srinivasan, and G. Swallow, "RSVP-TE: extensions to RSVP for LSP tunnels," IETF RFC 3209, 2001.

[5] B. Rajagopalan, J. Luciani, and D. Awduche, "IP over optical networks: a framework," IETF RFC 3717, 2004.

[6] B. Chen, S. Bose, W. Zhong, and H. Wang, "A new lightpath establishing method for dynamic traffic grooming under the overlay model," Photonic Network Commun., vol. 17, no. 1, pp. 11$20,2009$. 
[7] N. Sengezer and E. Karasan, "An efficient virtual topology design and traffic engineering scheme for IP/WDM networks," Lect. Notes Comput. Sci., vol. 4534, pp. 319-328, 2007.

[8] S. Azodolmolky, Y. Pointurier, M. Klinkowski, E. Marin, D. Careglio, J. Sole-Pareta, M. Angelou, and I. Tomkos, "On the offline physical layer impairment aware RWA algorithms in transparent optical networks: state-of-the-art and beyond," in Int. Conf. on Optical Network Design and Modeling, 2009.

[9] R. Ramaswami and K. Sivarajan, "Routing and wavelength assignment in all-optical networks," IEEE/ACM Trans. Netw., vol. 3, no. 5, pp. 489-500, 1995.

[10] H. Zang, J. Jue, and B. Mukherjee, "A review of routing and wavelength assignment approaches for wavelength-routed optical WDM networks," Opt. Networks Mag., vol. 1, no. 1, pp. 47-60, 2000.

[11] G. Agrawal, Fiber-Optic Communication Systems. WileyInterscience, 2002.

[12] G. Agrawal, Lightwave Technology: Communication Systems. New Jersey, USA: Wiley, 2005.

[13] I. Tomkos, D. Vogiatzis, C. Mas, I. Zacharopoulos, A. Tzanakaki, and E. Varvarigos, "Performance engineering of metropolitan area optical networks through impairment constraint routing," IEEE Commun. Mag., vol. 42, no. 8, pp. S40S47, 2004.

[14] K. Manousakis, K. Christodoulopoulos, and E. Varvarigos, "Impairment-aware offline RWA for transparent optical networks," in Proc. IEEE Conf. on Computer Communications (INFOCOM), 2009, pp. 1557-1565.

[15] K. Christodoulopoulos, K. Manousakis, and E. Varvarigos, "Considering physical layer impairments in offline RWA," IEEE Network, vol. 23, no. 3, pp. 26-33, 2009.

[16] X. Yang, L. Shen, and B. Ramamurthy, "Survivable lightpath provisioning in WDM mesh networks under shared path protection and signal quality constraints," J. Lightwave Technol., vol. 23, no. 4, pp. 1556-1567, 2005.

[17] M. Lima, A. Cesar, and A. Araujo, "Optical network optimization with transmission impairments based on genetic algorithm," in Proc. of the 2003 SBMO/IEEE MTT-S Int. Microwave and Optoelectronics Conf., 2003, vol. 1, pp. 361-365.

[18] D. Monoyios, K. Vlachos, M. Aggelou, and I. Tomkos, "On the use of multi-objective optimization algorithms for solving the impairment aware-RWA problem," in IEEE Int. Conf. on Communications, 2009.

[19] M. Ezzahdi, S. Al Zahr, M. Koubàa, N. Puech, and M. Gagnaire, "LERP: a quality of transmission dependent heuristic for routing and wavelength assignment in hybrid WDM networks," École Nationle Superieure des Telecommunications, Technical Report 2006D003, 2006.

[20] B. Ramamurthy, D. Datta, H. Feng, J. Heritage, and B. Mukherjee, "Impact of transmission impairments on the teletraffic performance of wavelength-routed optical networks," $J$. Lightwave Technol., vol. 17, no. 10, pp. 1713-1723, 1999.

[21] S. Zsigmond, B. Megyer, T. Cinkler, A. Tzanakaki, and I. Tomkos, "A new method for considering physical impairments in multilayer routing," in COST291/GBOU ONNA Workshop on Design of Next Generation Optical Networks, 2006.

[22] Y. Huang, J. Heritage, and B. Mukherjee, "Connection provisioning with transmission impairment consideration in optical WDM networks with high-speed channels," J. Lightwave Technol., vol. 23, no. 3, pp. 982-993, 2005.

[23] J. He, M. Brandt-Pearce, Y. Pointurier, C. Brown, and S. Subramaniam, "Adaptive wavelength assignment using wavelength spectrum separation for distributed optical networks," in IEEE Int. Conf. on Communications, 2007, pp. 2406-2411.
[24] X. Yang and B. Ramamurthy, "Dynamic routing in translucent WDM optical networks," in IEEE Int. Conf. on Communications, 2002, vol. 5, pp. 2796-2802.

[25] S. Azodolmolky, D. Klonidis, I. Tomkos, Y. Ye, C. Saradhi, E. Salvadori, M. Gunkel, K. Manousakis, K. Vlachos, E. Varvarigos, R. Nejabati, D. Simeonidou, M. Eiselt, J. Comellas, J. SolePareta, C. Simonneau, D. Bayart, D. Staessens, D. Colle, and M. Pickavet, "A dynamic impairment aware networking solution for transparent mesh optical networks," IEEE Commun. Mag., vol. 47, no. 5, pp. S38-S47, 2009.

[26] S. Azodolmolky, M. Klinkowski, E. Marin, D. Careglio, J. Pareta, and I. Tomkos, "A survey on physical layer impairments aware routing and wavelength assignment algorithms in optical networks," Comput. Netw., vol. 53, no. 7, pp. 926944, 2009.

[27] Y. Huang, W. Wen, J. Heritage, and B. Mukherjee, "Signalquality consideration for dynamic connection provisioning in all-optical wavelength-routed networks," Proc. SPIE, vol. 5285, pp. 163-173, 2003.

[28] C. Cantrell, "Transparent optical metropolitan-area networks," in LEOS, 16th Annu. Meeting, 2003, vol. 2, pp. 608-609.

[29] W. L. Winston, Operations Research: Applications and Algorithms, 4th ed., Thomson, 2004.

[30] "Dynamic Impairment Constraint Network for Transparent Mesh Optical Networks (DICONET) project," http:// www.diconet.eu.

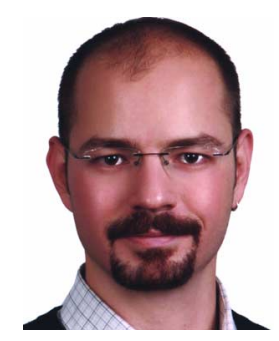
algorithms, and protocols.

Namik Sengezer received the B.S. and M.S. degrees in electrical engineering from Bilkent University, Turkey, in 2002 and 2004. He is currently working toward the Ph.D. degree in the Department of Electrical and Electronics Engineering. He has been participating in the FP6-IST Network of Excellence (NoE) e-Photon/ONe+ and FP7-IST NoE BONE projects. His research interests include design and planning of optical networks, traffic engineering, routing

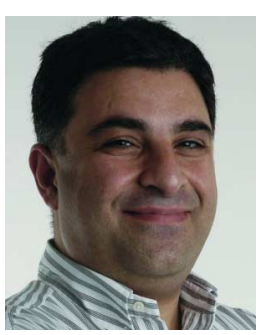

Ezhan Karasan (S'88-M'95) received the B.S. degree from Middle East Technical University, Ankara, Turkey; the M.S. degree from Bilkent University, Ankara, Turkey; and the Ph.D. degree from Rutgers University, Piscataway, NJ, all in electrical engineering, in 1987, 1990, and 1995, respectively. During 1995-1996, he was a Postdoctorate Researcher at Bell Labs, Holmdel, NJ. From 1996 to 1998, he was a Senior Technical Staff Member in the Lightwave Networks Research Department at AT\&T Labs-Research, Red Bank, NJ. He has been with the Department of Electrical and Electronics Engineering at Bilkent University since 1998, where he is currently an Associate Professor. He has been participating in the FP6-IST Network of Excellence (NoE) e-Photon/ONe+ and FP7-IST NoE BONE projects. His current research interests are in the application of optimization and performance analysis tools for the design, engineering, and analysis of optical networks and wireless ad hoc/mesh/sensor networks. Dr. Karasan is a member of the Editorial Board of Optical Switching and Networking. He was the recipient of the 2004 Young Scientist Award from the Turkish Scientific and Technical Research Council (TUBITAK), the 2005 Young Scientist Award from the Mustafa Parlar Foundation, and the Career Grant from TUBITAK in 2004. He received a fellowship from the NATO Science Scholarship Program for overseas studies in 19911994. 